\title{
Évaluation de l'état de santé écologique des hydrosystèmes par l'uti- lisation des traits biologiques
}

\author{
S. Charvet ${ }^{1}$ \\ M.C. Roger ${ }^{1}$ \\ B. Faesse $1^{1}$ \\ M. Lafont ${ }^{1}$
}

Mots-clés : cours d'eau, station d'épuration, pollution, macroinvertébrés, traits biologiques, approche fonctionnelle.

Les macroinvertébrés benthiques sont couramment utilisés dans le domaine de la bioindication. Pourtant les méthodes indicielles globales ne permettent pas de poser un diagnostic sur les causes réelles de dégradation. Cette étude propose donc d'intégrer les avancées théoriques récentes mettant en relation les stratégies biologiques des organismes (reproduction, durée de vie, habitudes alimentaires et respiratoires) avec les perturbations de leur environnement.

Cette approche a été appliquée à la Chalaronne, un cours d'eau soumis à des effluents d'une station d'épuration. L'analyse biocénotique, basée sur l'abondance des taxons, permet de séparer les stations amont des stations aval puisque 17,9\% de la variabilité totale du tableau de données y est consacrée. Au niveau fonctionnel, l'utilisation des traits biologiques définis pour chaque taxon permet une meilleure séparation amont - aval, puisque la valeur obtenue est de $23,1 \%$.

Cet exemple nous montre que la structure fonctionnelle observée en amont correspond à des caractéristiques de résilience. Au contraire la structure fonctionnelle observée en aval correspond à des caractéristiques de résistance.

L'approche fonctionnelle présente donc deux intérêts : d'une part elle permet une bonne discrimination des situations étudiées, d'autre part elle ouvre des perspectives intéressantes pour l'étude des fonctionnalités biologiques des écosystèmes.

\section{Biomonitoring of freshwater ecosystems by the use of biological traits}

Keywords : stream, waste water treatment plant, pollution, macroinvertebrates, biological traits, functional approach.

Benthic macroinvertebrates are of common use in bioindication. But global indices are not able to diagnose actual causes of damaging. The aim of this study is to integrate recent developments linking biological strategies of animals (reproduction, life duration, feeding habits, respiration) with environmental disturbance.

This methodology has been applied to the Chalaronne river, a water body receiving effluents from a waste water treatment plant. Biocenotic analysis, based on abundances of taxa, allows discrimination of upstream from downstream stations since $17.9 \%$ of the variability is dedicated to it. Functional analysis, based on biological traits, gives a better discrimination of stations, since the value is equal to $23.1 \%$.

This study shows that functional structure observed upstream is characteristic of resilience. On the contrary, functional structure observed downstream is characteristic of resistance.

A functional approach is interesting for two reasons : first as a mean to discriminate stations efficiently, and secondly it is promising in study of biological functionalities of ecosystems.

\section{Introduction}

Les écosystèmes aquatiques sont peuplés d'organismes végétaux et animaux dont la distribution dépend des conditions environnementales. Ainsi, tout changement des conditions environnementales entraî-

1. Cemagref, Laboratoire Diagnose des Systèmes Aquatiques, 3 bis quai Chauveau, F-69336 Lyon Cedex 09, France. ne des changements dans les communautés, notamment au niveau de la composition faunistique (Hynes 1963, Macan 1974, Mason 1981, Chapman 1992). Parmi les méthodes utilisant les organismes aquatiques afin de mettre en évidence d'éventuelles altérations environnementales, certaines sont basées sur la prise en considération d'espèces de macroinvertébrés benthiques (Woodiwiss 1964, Tufféry \& Verneaux 1967, Washington 1984, Lafont 1989). Ces organismes, de par la diversité des formes taxinomiques et fonction- 
nelles qu'ils représentent, leur durée de vie, leur distribution dans l'ensemble des eaux courantes, constituent des bioindicateurs potentiels. Pourtant les méthodes globales existantes, indices biotiques ou indices de diversité, ne permettent pas de poser un diagnostic sur les causes réelles de dégradation, et intègrent mal les fluctuations naturelles, temporelles et spatiales des communautés vivantes. Ces méthodes ne prennent généralement en considération que la présence, l'abondance ou la biomasse des taxons.

Cependant, depuis quelques années, certains auteurs ont préconisé de retenir la biologie des espèces, en plus des variables -présence-abondance-biomasse, pour essayer de mieux comprendre le fonctionnement des écosystèmes, incluant l'impact des pollutions (Southwood 1988, Townsend \& Hildrew 1994). De plus, le développement d'un nouveau type d'outil, le codage flou (Chevenet et al. 1994, Statzner et al. 1994b), permet d'attribuer aux différentes espèces rencontrées une valeur quantifiant l'affinité que portent ces espèces pour les différentes modalités d'une variable donnée. C'est ainsi que les connaissances biologiques peuvent être formalisées, chaque variable étant appelée trait biologique. Ensuite, par couplage du tableau espècesabondances et du tableau espèces-traits, il est possible d'obtenir une image fonctionnelle d'un milieu.

Cette nouvelle approche par les traits biologiques a été testée sur une étude d'impact de pollution, et comparée à une approche classique utilisant seulement les abondances des taxons. De plus, il nous paraissait intéressant de comparer les résultats obtenus par cette approche avec les prédictions de certains auteurs concernant les modèles théoriques des traits d'histoire de vie (Southwood 1988, Townsend \& Hildrew 1994).

\section{Site étudié, matériel et méthodes}

\subsection{Présentation du site}

Le milieu étudié est la Chalaronne, affluent rive gauche de la Saône qui coule à $50 \mathrm{~km}$ au nord de Lyon. Cette rivière reçoit les effluents de la station d'épuration (STEP) de Châtillon-sur-Chalaronne. Deux sites ont été prospectés dans cette étude : le premier est situé en amont de la station d'épuration et reçoit les déversoirs d'orage de la ville de Châtillon-sur-Chalaronne, alors que le deuxième est situé en aval de la station d'épuration dont il reçoit les effluents.

\subsection{Protocole d'échantillonnage}

Pour chaque station, treize campagnes de prélèvements ont été réalisées : la campagne numéro 1 correspond au $2 / 3 / 95$, la 2 au 22/3/95, la 3 au $5 / 5 / 95$, la 4 au $7 / 6 / 95$, la 5 au 25/6/95, la 6 au 20/7/95, la 7 au 24/8/95, la 8 au 27/9/95, la 9 au 19/10/95, la 10 au 23/11/95, la 11 au 21/12/95, la 12 au 25/1/96, et la 13 au 22/2/96.

Des échantillons d'eau ont été prélevés à chaque date afin de mesurer les paramètres physico-chimiques suivants : température (temp), conductivité (cond, à $20^{\circ} \mathrm{C}$, NF EN 27888), $\mathrm{pH}(\mathrm{pH}, \mathrm{NF} \mathrm{T} 90-008)$, oxygène dissous (O2, NF EN 25813), matières en suspension (MES, NF EN 872), carbone organique total (COT, NF T 90-102), carbone organique dissous (COD, NF T 90102), sels ammoniacaux (NH4, NF T 90-015), nitrites (NO2, NF EN ISO 13395), nitrates (NO3, NF EN ISO 13395), orthophosphates (PO4, NF EN 1189) et phosphore total (P tot, NF EN 1189).

Les treize campagnes de prélèvements de macroinvertébrés ont été réalisées conformément au protocole de la norme de l'indice biologique IBGN (AFNOR 1992) : huit échantillons ont été effectués dans huit habitats distincts, afin d'obtenir une vision représentative du milieu étudié. Dans les habitats lotiques, cinq prélèvements ont été réalisés à l'aide d'un filet de type Surber (maille de $500 \mu \mathrm{m}$, surface de $0 ; 1 \mathrm{~m}^{2}$ ), alors que dans les habitats lentiques, trois prélèvements ont été effectués à l'aide d'un filet Troubleau.

Les organismes ont été identifiés au genre ou à l'espèce (Trichoptères, Ephéméroptères, Coléoptères, Odonates, Mégaloptères, Crustacés, Mollusques, Achètes).

\section{Traitement des données}

Les données physico-chimiques ont été traitées à l'aide d'une Analyse en Composantes Principales Normée, ou ACPN (Hotelling 1933), particulièrement adaptée à l'étude des structures entre les variables (Dolédec \& Chessel 1991).

Les données portant sur les effectifs des genres et des espèces ont été traitées à l'aide d'une Analyse Factorielle des Correspondances, ou AFC (Benzécri 1973a, 1973b), adaptée aux tableaux de contingences, et donnant une représentation des éventuelles relations entre deux variables nominales : les lignes et les colonnes (Lebart et al. 1995).

Les traits biologiques des genres et des espèces (Dolédec \& Statzner 1994, Statzner et al. 1994a) sont décrits dans le tableau 1. Ces traits biologiques sont pondérés par les abondances, et le tableau en codage flou qui en résulte est traité par une Analyse des Correspondances Floues, ou ACF. L'ACF est une Analyse des Correspondances Multiples (ACM) étendue à des tableaux qui ne sont pas disjonctifs complets (Chevenet et al. 1994). 
Tableau 1. Description des traits biologiques (le texte en gras représente les variables, le texte non gras représente les modalités des variables).

Table 1. Description of biological traits (text in bold represents variables, non-bold text represents modalities of variables).

(1) Taille

$\leq 5 \mathrm{~mm} \quad>5-10 \mathrm{~mm} \quad>10-20 \mathrm{~mm} \quad>20-40 \mathrm{~mm} \quad>40 \mathrm{~mm}$

(2) Nombre de descendants par cycle reproducteur

$\leq 100>100-1000 \quad>1000-3000 \quad>3000$

(3) Nombre de cycles reproducteurs par an

$\begin{array}{llll}<1 & =1 & =2 & >2\end{array}$

(4) Nombre de cycles reproducteurs par individu

$\leq 1 \quad 2 \quad>2$

(5) Durée de vie adulte

$\leq 1$ jour $\quad>1-10$ jours $\quad>10-30$ jours $\quad>30-90$ jours $>90-365$ jours $>365$ jours

(6) Technique de reproduction

individu seul mâle et femelle

(7) Période de reproduction

Mars Avril Mai Juin Juillet Août Septembre Octobre Novembre

(8) Soin parental

ovoviviparité oeufs isolés oeufs aquatiques cimentés oeufs dans la végétation oeufs terrestres cimentés bourgeonnement, régénẹration

(9) Potentialités de dissémination (eau)

$\leq 10 \mathrm{~m} \quad>10-100 \mathrm{~m} \quad>100-1000 \mathrm{~m} \quad>1000 \mathrm{~m}$

(10) Attachement au sol/substrat

nageurs rampeurs creuseurs attachement temporaire attachement permanent

\begin{tabular}{|c|c|c|c|}
\hline \multicolumn{4}{|c|}{ (11) Flexibilité du corps } \\
\hline élevée $\left(>45^{\circ}\right)$ & $\left(>10-45^{\circ}\right.$ & aucune $\left(<10^{\circ}\right)$ & \\
\hline \multicolumn{4}{|l|}{ (12) Forme du corps } \\
\hline hydrodynamique & aplatie & cylindrique & sphérique \\
\hline \multicolumn{4}{|c|}{ (13) Habitudes alimentaires (larves) } \\
\hline déchiqueteurs & racleurs & récolteurs & filtreurs \\
\hline \multicolumn{4}{|l|}{ (14) Nourriture } \\
\hline détritus $\leq 1 \mathrm{~mm}$ & \multicolumn{2}{|c|}{ détritus $>1-10 \mathrm{~mm}$} & détritus $>10 \mathrm{~mm}$ \\
\hline plantes : microphytes & \multicolumn{2}{|c|}{ plantes : algues } & plantes : macrophytes \\
\hline $\operatorname{animaux} \leq 1 \mathrm{~mm}$ & \multicolumn{2}{|c|}{ animaux $>1-10 \mathrm{~mm}$} & animaux $>10 \mathrm{~mm}$ \\
\hline
\end{tabular}

(15) Respiration (aquatique : aqua ; aérienne : aér)

aqua (plastron) aér (spiracle) aqua (spiracle) aqua (branchies) aqua (tégument) 
Les analyses multivariées ont toutes été réalisées à l'aide du logiciel ADE-4 (Chessel \& Dolédec 1992). Cependant des tests non-paramétriques ont dû être effectués afin de déterminer quels taxons et quels traits biologiques étaient significativement différents entre l'amont et l'aval. De plus une analyse de variance a permis de comparer les informations apportées par les abondances et par les traits biologiques sur la base des distances séparant les stations à chaque campagne. Les deux dernières analyses statistiques ont nécessité l'utilisation du logiciel Statistica 4.1 (Statsoft 1997).

\section{Résultats}

L'ACPN réalisée sur les données physico-chimiques permet d'extraire sur le premier axe factoriel $44,6 \%$ de la variabilité totale du nuage de points, tandis que le deuxième axe représente $16,9 \%$ de la variabilité (Fig.
1). Les variables significatives ( $p<0,05$ ) sur $F 1$ sont la conductivité (cond), les sels ammoniacaux (NH4), les nitrites (NO2), les orthophosphates (PO4), le phosphore total ( $\mathrm{P}$ tot) et le carbone organique dissous (COD). Les variables significatives sur $\mathrm{F} 2$ sont la température (temp) et le carbone organique total (COT). La part de discrimination obtenue par analyse inter-classes entre les stations amont et les stations aval vaut $3,1 \%$.

L'AFC effectuée sur le tableau des abondances spécifiques (Tableau 2) attribue $22,5 \%$ de la variabilité au premier axe factoriel, et $19,1 \%$ au deuxième, soit $41,6 \%$ pour les deux axes (Fig. 2). Le pourcentage de discrimination entre les stations amont et aval est égal à $17,9 \%$. Les espèces les plus fréquemment rencontrées dans les échantillons amont sont Hydropsyche pellucidula, $H$. exocellata, $H$. siltalai, Psychomyia pusilla, Hydroptila sparsa, Allogamus auricollis, Limne-

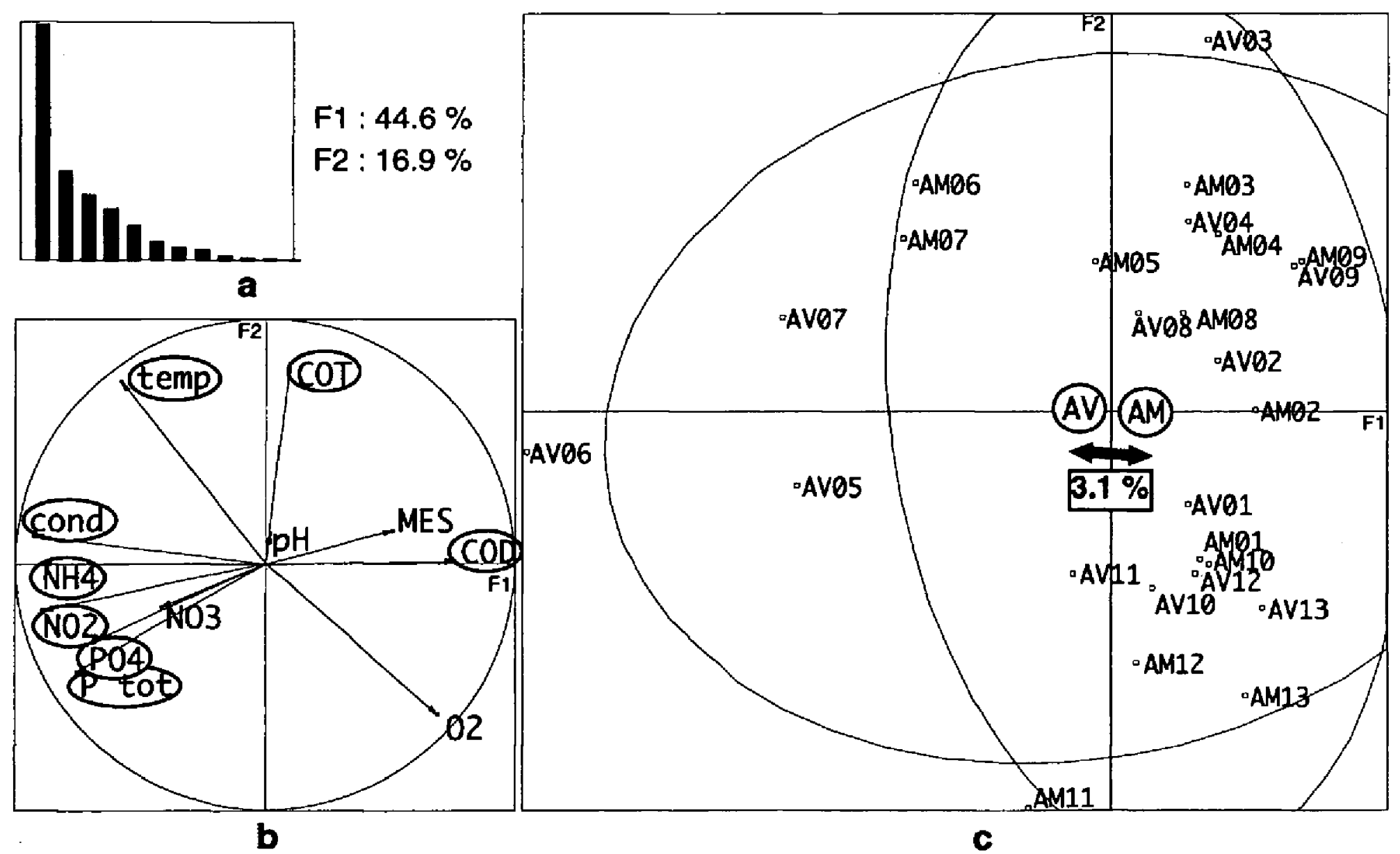

Fig. 1. Analyse en Composantes Principales Normée des données physico-chimiques. a : graphe des valeurs propres. $\mathbf{b}:$ carte factorielle des variables physico-chimiques intervenant dans l'analyse (les variables significatives sont entourées); temp = température ; cond = conductivité $; \mathrm{O} 2=$ oxygène dissous $; \mathrm{MES}=$ matières en suspension $; \mathrm{COT}=$ carbone organique total $; \mathrm{COD}=\mathrm{carbone}$ organique dissous $; \mathrm{NH} 4=$ sels ammoniacaux $; \mathrm{NO} 2=$ nitrites $; \mathrm{NO} 3=$ nitrates $; \mathrm{PO} 4=$ orthophosphates $; \mathrm{P}$ tot $=$ phosphore total. $\mathrm{c}$ : carte factorielle des échantillons (AM et AV sont les centres de gravité des ellipses des échantillons amont et aval, numérotés de 01 à 13).

Fig. 1. Standardized Principal Component Analysis on physico-chemical data. $\mathbf{a}$ : graphic of eigenvalues. $\mathbf{b}$ : factor map of physicochemical variables (significant variables are enclosed); temp = temperature ; cond = conductivity; $\mathrm{O} 2=$ dissolved oxygen MES $=$ suspended matter $; \mathrm{COT}=$ total organic carbon; $\mathrm{COD}=$ dissolved organic carbon; $\mathrm{NH} 4=$ ammonium $; \mathrm{NO} 2=$ nitrite $; \mathrm{NO} 3=$ nitrate ; PO4 = orthophosphate ; $\mathrm{P}$ tot $=$ total phosphonus. $\mathrm{c}:$ factor map of samples (AM and AV are gravity centers of the two ellipses of upstream and downstream samples, numbered 01 to 13). 
philus, Halesus, H. digitatus, Mystacides azurea, Cloeon dipterum, Habrophlebia, Elmis, Limnius volckmari, Oulimnius tuberculatus, Esolus, Hydraena riparia, Platycnemis, Sialis lutaria, Gammarus, Physella acuta, Lymnaea palustris, Bathyomphalus contortus, Armiger crista, Menetus dilatatus, Pisidium henslowanum, tandis que les espèces les plus fréquemment rencontrées dans les échantillons aval sont $\mathrm{Ti}$ nodes waeneri, Halesus radiatus, Calopteryx virgo, Asellus aquaticus, Orconectes limosus, Valvata cristata, V. piscinalis, Bithynia tentaculata, Lymnaea peregra, Ferissia wautieri, Ancylus lacustris, Sphaerium corneum, Glossiphonia, Erpobdella, Helobdella stagnalis, Hemiclepsis marginata, Piscicola geometra.

L'ACF exécutée sur le tableau obtenu par pondération des traits biologiques par les abondances des taxons (Tableau 3) donne 35,7\% de la variabilité du tableau de données au premier axe factoriel, et $26,9 \%$ au deuxième, soit $62,6 \%$ pour les deux (Fig. 3). Le pourcentage de discrimination entre les stations amont et aval est égal à $23,1 \%$. Un test basé sur la somme des rangs (Mann - Whitney : U) et un test basé sur les rangs signés (Wilcoxon: W) montrent que 11 des 15 variables sont à même de séparer de façon significative les stations amont des stations aval : taille, nombre de descendants par cycle reproducteur, nombre de cycles reproducteurs par an, nombre de cycles reproducteurs par individu, durée de vie adulte, potentialités de dissémination (eau), attachement au sol/substrat, période reproductive, habitudes alimentaires (larves), nourriture, et respiration (Fig. 3).

Dans notre étude, les échantillons amont sont donc constitués de macroinvertébrés benthiques possédant une petite taille, un grand nombre de descendants par cycle reproducteur et un grand nombre de cycles reproducteurs par an, mais seulement un petit nombre de cycles reproducteurs par individu. Leur durée de vie est courte, ils sont récolteurs ou filtreurs, ils se nourrissent de petits détritus ou de macrophytes, et respirent à 1'aide de plastrons ou de branchies.

A l'inverse, les échantillons aval sont composés d'organismes ayant une grande taille, un petit nombre de descendants par cycle reproducteur et un petit nombre de cycles reproducteurs par an, mais un grand nombre de cycles reproducteurs par individu. Leur durée de vie est longue, ce sont des perceurs de petits animaux, et respirent grâce à leur tégument.

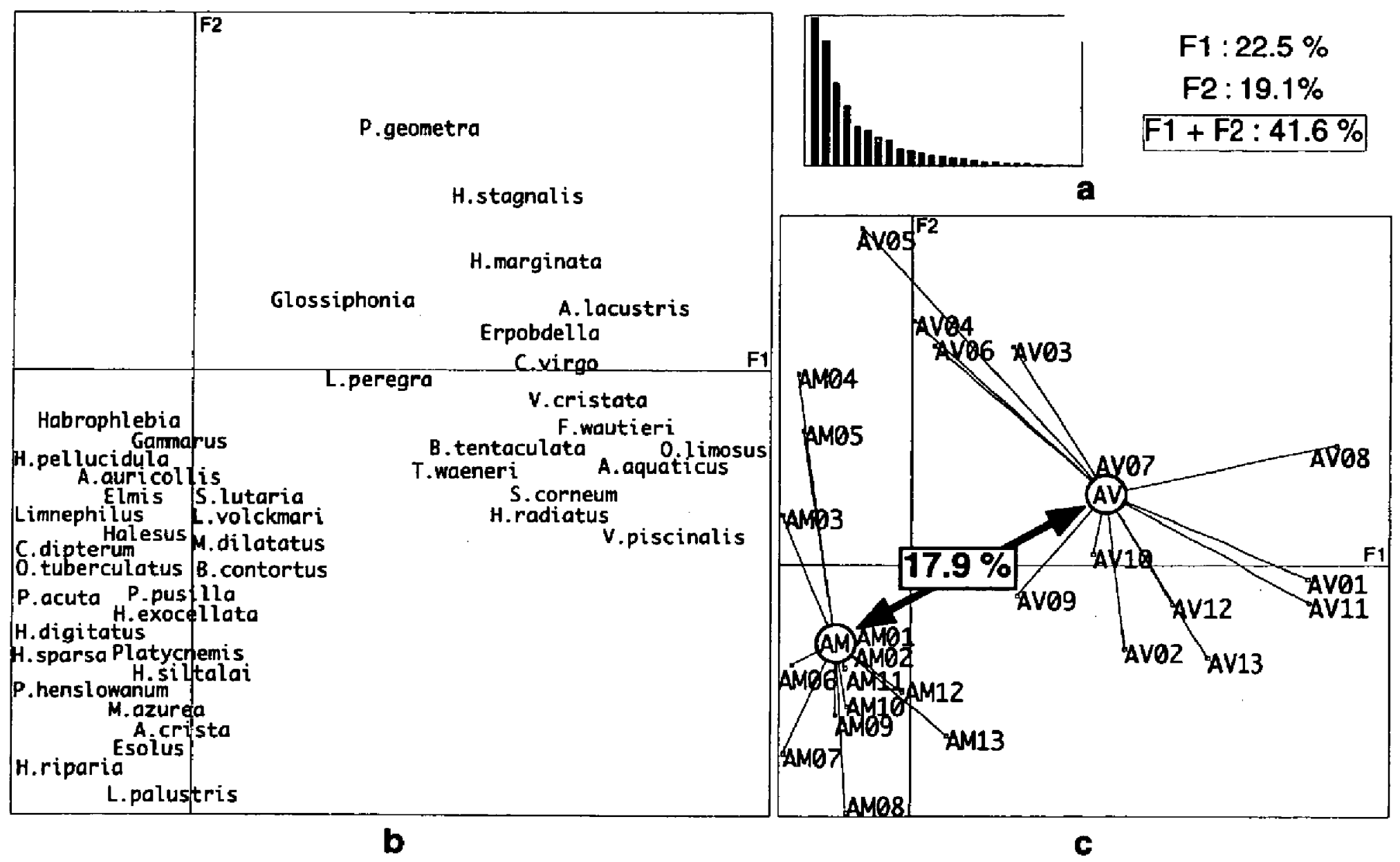

Fig. 2. Analyse Factorielle des Correspondances réalisée sur le tableau des abondances spécifiques. a : graphique des valeurs propres. b : carte factorielle des espèces. $\mathbf{c}$ : carte factorielle des échantillons (AM et AV sont les centres de gravité des nuages des échantillons amont et aval, numérotés de 01 à 13 ).

Fig. 2. Correspondence Analysis performed on species abundances array. $\mathbf{a}$ : graphic of eigenvalues. $\mathbf{b}$ : factor map of species. $\mathbf{c}:$ factor map of samples (AM and AV are gravity centers of the two stars of upstream and downstream samples, numbered 01 to 13 ). 
Tableau 2. Abondance (tous prélèvements confondus) des 62 taxons dans les 13 échantillons amont (AM01 à AM13) et dans les 13 echantillons aval (AV01 à AV13).

Table 2. Abundance (all habitats mixed) of 62 taxa at 13 upstream samples (AM01 to AM|.3) and at 13 downstream samples (AV01 to AV13).

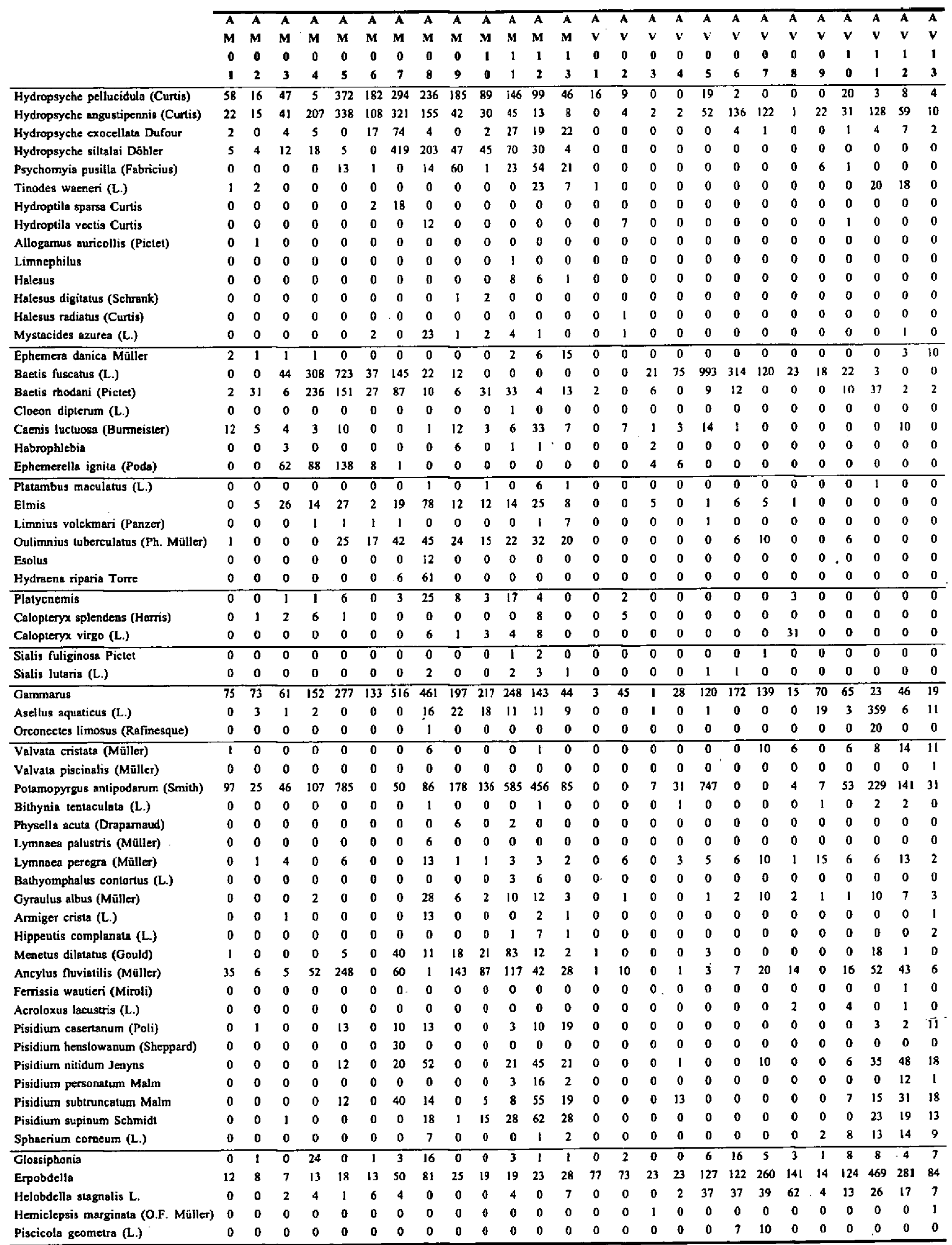


Tableau 3. Distribution des 74 modalités des 15 variables des traits biologiques dans les 13 echantillons amont (AM01 à AM13) et dans les 13 échantillons aval (AV01 à AV13). Les variables et les modalites correspondent à la description des traits biologiques réalisée dans le tableau 1 . Toutes les valeurs sont multipliées par 100 .

Table 3. Distribution of 74 modalities of 15 variables of the biological traits at 13 upstream samples (AM01 to AM13) and at 13 downstream samples (AV01 to AV13). Variables and modalities correspond to the description of biological traits shown in table 1 . All values are multiplied by 100 .

\begin{tabular}{|c|c|c|c|c|c|c|c|c|c|c|c|c|c|c|c|c|c|c|c|c|c|c|c|}
\hline & & $\begin{array}{c}\mathbf{A} \\
\mathbf{M} \\
\mathbf{0} \\
\mathbf{1}\end{array}$ & $\begin{array}{c}\mathbf{A} \\
\mathbf{M} \\
\mathbf{2}\end{array}$ & $\begin{array}{c}\hat{\mathbf{A}} \\
\mathbf{M} \\
\mathbf{0} \\
\mathbf{3}\end{array}$ & $\begin{array}{c}\text { A } \\
M \\
0 \\
4\end{array}$ & $\begin{array}{c}\mathbf{A} \\
\mathrm{M} \\
\mathbf{0} \\
\mathbf{5}\end{array}$ & $\begin{array}{c}A \\
M \\
0 \\
6\end{array}$ & $\begin{array}{c}A \\
M \\
0 \\
7\end{array}$ & $\begin{array}{c}A \\
M \\
0 \\
g\end{array}$ & $\begin{array}{c}\mathbf{A} \\
\mathbf{M} \\
0 \\
9\end{array}$ & $\begin{array}{c}\hat{A} \\
\mathbf{M} \\
1 \\
0\end{array}$ & $\begin{array}{c}A \\
M \\
1 \\
1\end{array}$ & $\begin{array}{c}A \\
M \\
1 \\
2\end{array}$ & $\begin{array}{c}A \\
M \\
1 \\
3\end{array}$ & $\begin{array}{l}\mathbf{A} \\
\mathbf{v} \\
\mathbf{1}\end{array}$ & $\begin{array}{l}\boldsymbol{A} \\
\boldsymbol{V} \\
\mathbf{0} \\
\mathbf{2}\end{array}$ & $\begin{array}{l}A \\
\mathbf{v} \\
0 \\
\mathbf{3}\end{array}$ & $\begin{array}{l}A \\
\mathbf{V} \\
0 \\
4\end{array}$ & $\begin{array}{l}\hat{A} \\
\mathbf{0} \\
0 \\
5\end{array}$ & $\begin{array}{l}A \\
\mathbf{v} \\
6 \\
6\end{array}$ & $\begin{array}{l}\mathbf{A} \\
\mathbf{v} \\
\mathbf{0} \\
7\end{array}$ & $\begin{array}{l}A \\
\mathbf{V} \\
0 \\
\mathbf{B}\end{array}$ & $\begin{array}{l}1 \\
v \\
0 \\
9\end{array}$ \\
\hline Vartable 1 & modallte 1 & 20.2 & 11.1 & 17.9 & 12.6 & 23.1 & 5.5 & 11.02 & 21.51 & 16,7 & 19.83 & 33.8 & 42.0 & 39.5 & 1.0 & 4.72 & 20.12 & 27.83 & 33.01 & 10.78 & 8.4 & 4.9 & 4.8 \\
\hline & 2 & 14.5 & 11.7 & & 34.0 & 33.4 & 8.2 & B.7 & 7.71 & 16.5 & 9.11 & 18.5 & 24,5 & 19.2 & 1.2 & 4.53 & 36.04 & 40.54 & 49.52 & 28.31 & 13.0 & 7.41 & 13.6 \\
\hline & & 43.1 & 42.5 & 34,9 & 40.5 & 36.1 & 65.9 & 60.6 & 42.34 & 46,6 & 43.93 & 33.1 & 20.02 & 29.1 & 19.3 & 22.61 & $10.5 ?$ & 7.87 & 7.02 & 29.23 & 30.12 & $26.3 \mathrm{~J}$ & 30.2 \\
\hline & $t \leqslant 4$ & 21.5 & 34.4 & 15.2 & 12.5 & 7.4 & 20.4 & 19.32 & 28.32 & 20.1 & 26.81 & 15.4 & 12.6 & 18.2 & 78.5 & 66.13 & 33.42 & $23.9 \mathrm{~J}$ & 10.53 & 31.84 & $48.5 \mathrm{~s}$ & 53.95 & 51.4 \\
\hline & condulles 5 & 0.0 & 0.4 & 0.4 & 0.4 & 0.0 & 0.0 & 0.0 & 0.3 & 0.1 & 0.3 & 0.2 & 0.9 & 0.0 & 0.0 & 2.20 & 0.00 & 0.00 & 0.0 & 0.00 & 0.0 & 7.50 & 0.0 \\
\hline Varluble 2 & modalitt I & 68.1 & 60.3 & 40.2 & 29.5 & 44.9 & 31.1 & 39.75 & 58.56 & 60.3 & 69.87 & 74.5 & 74.56 & 68.0 & 81.2 & 80.25 & 50.05 & 54.54 & 48.7 & 44.86 & 68.48 & 81.26 & 63.9 \\
\hline & modatite 2 & 30.8 & 25.8 & 39.8 & 26.7 & 26.7 & 57.5 & 50.53 & 39.73 & 37.8 & 26.72 & 23.5 & 24.72 & 26.6 & 17.1 & 19.81 & 11.24 & 4.64 & 4.11 & 17.11 & 16.11 & 11.32 & 26.1 \\
\hline & Itt 3 & 0.3 & 6.7 & 19.1 & 35.6 & 26.4 & 9.3 & 8.1 & 1.5 & 1.7 & 1.7 & 1.0 & 0.2 & 1.2 & 0.8 & 0.03 & 35.44 & 41.04 & 47.13 & 37.51 & 15.5 & 7.4 & 10.0 \\
\hline & odalitt 4 & 0.9 & $1+2$ & 0.9 & B.2 & 2.0 & 2.1 & 1.7 & 0.2 & 0.3 & $1 . J$ & 1.0 & 0.6 & 4.3 & 0.8 & 0.03 & 3.50 & $0.0 \quad 0$ & 0.2 & 0.60 & 0.0 & 0.0 & 0.0 \\
\hline Variable 3 & odallte 1 & 0.5 & 0.5 & 0.2 & 0.5 & 0.0 & 0.0 & 0.0 & 0.4 & 0.0 & 0.0 & 0.3 & 0.8 & 2.6 & 0.0 & 0.30 & 0.0 & 0.50 & 0.1 & 0.80 & 0.6 & 0.2 & 0.7 \\
\hline & $t \leqslant 2$ & 41.0 & 30.6 & 47.7 & 38.5 & 39.4 & 23.5 & 44.2 & 48.04 & 43.6 & 44.15 & 51.3 & 49.0 & 46.3 & 78.6 & $60.1 \mathrm{~s}$ & 57.03 & 32.12 & 29.43 & 31.55 & 55.56 & 66.62 & 24.4 \\
\hline & dulitt 3 & 35.4 & 27.6 & 35.5 & 44.9 & 50.9 & 51.7 & 32.12 & 23.93 & 34.72 & 24.13 & 31.6 & 38.13 & 39.2 & 18.0 & 13.63 & 38.75 & 52.56 & 64.84 & 47.22 & 25.82 & 28.32 & 24.3 \\
\hline & modaltte 4 & 23.1 & 41.3 & 16.6 & 16.1 & 9.6 & 24.8 & 23.72 & 27.72 & 21.73 & 31.71 & 16.8 & 2.1 & 11.9 & 3.4 & 26.04 & 4.31 & $14.8 \mathrm{~S}$ & 5.72 & 20.51 & 18.0 & 4.95 & 50.6 \\
\hline Varfable 4 & modalite I & 40.6 & 41.3 & 62.6 & 73.8 & 62.1 & 69.8 & 67.3 & 50.05 & 5 & & 38.9 & 37.9 & 46.6 & 20.5 & 25.65 & 50.34 & 49.65 & 51.65 & 57.23 & 38.22 & 24.72 & 26.0 \\
\hline & dulthe 2 & 35.4 & 19.3 & & 13.1 & & 5.7 & 9.22 & & & & 44.3 & & & & & 39.23 & 32.54 & 41,31 & 18.53 & $35.2 \mathrm{~s}$ & $5 B .82$ & 21 . \\
\hline & otnithe 3 & 23.9 & 39.3 & 16.7 & 13.1 & 8.8 & 24.5 & 23.52 & 29.02 & 22 & 31.51 & 16.8 & 12.5 & 12.9 & 22.0 & 36.91 & $10.5 \mathrm{I}$ & 17.97 & 7.12 & 24.32 & 26.61 & 16.55 & 52.6 \\
\hline Variable 5 & modallté 1 & 3.9 & 7.7 & 14.8 & 26.8 & 20.0 & 7.0 & 6.1 & 1.2 & 2.6 & 1.8 & 1.1 & 2.7 & 2.3 & 0.7 & 4.02 & 28.33 & 32.13 & 36.02 & 28.31 & 11.7 & 5.6 & 7.5 \\
\hline & modallte 2 & 7.9 & 16.0 & 23.5 & 28.8 & 18.0 & 20.4 & 17.11 1 & 11.31 & 12.2 & 8.57 & 7.4 & 8.4 & 13.4 & 6.0 & 5.31 & $18.3 \mathrm{I}$ & 12.61 & $12.8 ;$ & 14.37 & 7.9 & 1.9 & B.1 \\
\hline & Itt 3 & 20.1 & 14.1 & 21.0 & 14.4 & 17.1 & 41.6 & 37.22 & 27.92 & 22.6 & $17.2 \mathrm{I}$ & 15.6 & 12.0 & 14.0 & 12.1 & 9.62 & 2.00 & 0.82 & 2.51 & 12.31 & 11.9 & 6.2 & 10.0 \\
\hline & dullite 4 & 0.0 & 0.4 & 0.3 & 0.2 & 0.0 & 0.0 & 0.0 & 0.30 & 0.4 & 0.4 & 0.7 & 1.3 & 0.3 & 0.0 & 1.80 & 0.0 & 0.00 & 0.0 & 0.10 & 0.1 & 5.0 & 0.0 \\
\hline & dalut $s$ & 11.4 & 6.1 & 7.9 & 5.3 & 10.1 & 2.7 & 9.4 & 15.92 & 20.1 & 19.51 & 18.1 & 19.12 & 24.7 & 2.0 & 9.86 & 6.8 & 6.70 & 0.6 & 3.69 & 9.0 & 6.91 & 11.7 \\
\hline & modalitie 6 & 56.7 & 55.7 & 32.5 & 24.4 & 34.8 & 18.4 & 30.2 & 43.44 & $42.0 \mathrm{~s}$ & 52.75 & 57.1 & 56.5 & 45.3 & 79.2 & 69.44 & 44.64 & 47.84 & 48.14 & 41.15 & 59.47 & 74.4 .6 & 62.8 \\
\hline rint & modalitit 1 & 42.0 & 17.2 & & 14.7 & & 0.4 & 11.11 & & & & & & & & 10.81 & & & & & 7.8 & 9.41 & \\
\hline ruble 7 & & $\frac{58.0}{4.8}$ & & 80 & $\frac{85.3}{2.2}$ & & 99.6 & & & & & & & & & & & & & & & & \\
\hline & $\begin{array}{l}\text { debite } 1 \\
\text { dalitu } 2\end{array}$ & $\begin{array}{l}4.8 \\
6.7\end{array}$ & $\begin{array}{l}4.9 \\
6.9\end{array}$ & $\begin{array}{l}2.7 \\
4.0\end{array}$ & $\begin{array}{l}2.2 \\
4.3\end{array}$ & $\begin{array}{l}2.7 \\
4.8\end{array}$ & $\begin{array}{l}2.6 \\
3.5\end{array}$ & $\begin{array}{l}2.5 \\
4.5\end{array}$ & $\begin{array}{l}3.7 \\
6.97\end{array}$ & $\begin{array}{l}3.6 \\
7.4\end{array}$ & $\begin{array}{l}4,4,6 \\
7.6\end{array}$ & $\begin{array}{l}4,5 \\
7.3\end{array}$ & $\begin{array}{l}4.1 \\
8.6\end{array}$ & $\begin{array}{l}3.6 \\
9.8\end{array}$ & $\begin{array}{l}13.0 \\
13.2\end{array}$ & $\begin{array}{ll}9.4 & 6 \\
11.17 & 7\end{array}$ & 7.68 & $\begin{array}{l}4.7 \\
6.3\end{array}$ & $\begin{array}{l}4.4 \\
4.6\end{array}$ & $\begin{array}{ll}5.4 & 8 \\
6.0 & 9\end{array}$ & $\begin{array}{ll}8.5 & 1 \\
9.9 & 1\end{array}$ & 12.6 & $\begin{array}{l}6.6 \\
9.0\end{array}$ \\
\hline & Libite 3 & 14.7 & 13.7 & $\begin{array}{l}4.0 \\
10.6\end{array}$ & $\begin{array}{l}4.3 \\
14.2\end{array}$ & 14.2 & 11.6 & 15.01 & 13.11 & $18.4 \mathrm{I}$ & I8.5 I & 18.1 & 13.6 & 15.9 & 16.4 & $15.6 \mathrm{I}$ & $14.5 \mathrm{I}$ & 13.51 & 12.61 & $14.6 \mathrm{l}$ & $15.3 \mathrm{l}$ & 16.0 & 13.3 \\
\hline & 164 & 18.9 & 17.1 & 27.2 & 22.2 & 21.2 & 22.5 & 23.02 & 20.72 & 20.6 & 18.51 & 15.5 & 16.6 & 19.0 & 1B.3 & 18.12 & 21.31 & $19.0 \mathrm{~J}$ & 16.92 & $20.1 \mathrm{l}$ & 19.01 & 18.0 & 15.8 \\
\hline & ine 5 & 14.2 & 14.3 & 19.2 & 15.0 & 14.7 & 21.1 & 21.42 & 21.11 & 15.11 & 14.31 & 12.9 & 14.3 & 15.9 & 17.8 & $16.0 \mathrm{~J}$ & 16.41 & 14.11 & 13.01 & 16.91 & 17.31 & 17.1 & 15.0 \\
\hline & dellitis 6 & 17.7 & 15.8 & 18.7 & 16.9 & 18.5 & 20.9 & 17.0 & 17.71 & 16.31 & 15.21 & 19.3 & 18.4 & 16.9 & 17.9 & $15.7 \mathrm{t}$ & 16.11 & 16.91 & 18.21 & 16.71 & 12.01 & 17.4 & 16.4 \\
\hline & 47 & 12.9 & 13.0 & 9.9 & 12.6 & 13.6 & 10.4 & 9.3 & 9.91 & 10.71 & 11.11 & 14.1 & 15.5 & 12.4 & 2.3 & 7.59 & 9.21 & $13.6 \mathrm{I}$ & $16.4 \mathrm{I}$ & 10.26 & 6.9 & 4.9 & 11.6 \\
\hline & & 5.8 & 9.2 & 4.4 & 7.7 & 5.7 & 4.7 & 4.5 & 4.14 & 4.8 & 6.15 & 5.7 & 5.2 & 4.1 & 0.7 & 4,44 & 4.96 & 6.47 & $2.1=$ & 5.73 & 3.5 & 1.3 & 7.3 \\
\hline & modallite g & 4.2 & 5.1 & 3,3 & 4,8 & 4.7 & 2,8 & 2.8 & $2,8 \quad 3$ & 3.2 & 4,2 & 4,4 & 3.8 & 2.5 & 0.4 & 2,33 & 3,65 & 5.56 & $6.8 \quad 4$ & 4,62 & 2.7 & 1.1 & 30 \\
\hline Vartable 8 & modalite 1 & 52.8 & $5 t .8$ & 28.6 & 22.8 & 34.5 & 24.1 & 29.73 & 39.03 & 39.35 & 51.45 & 57.7 & 62.2 & 47.6 & 3.02 & 27.21 & 12.23 & 38.64 & 40.52 & 22.11 & 19.97 & 7.15 & 55.03 \\
\hline & tite 2 & 3.1 & 2.1 & 8.7 & 8.1 & 7.0 & 2.0 & 1.6 & 0.41 & 1.8 & $0.3 \mathrm{D}$ & 0.6 & 2.5 & 2.8 & 0.0 & 3.01 & $12.2 \mathrm{I}$ & 11.91 & 12.2 & 9.44 & 4.0 & 1.9 & 2.5 \\
\hline & te 3 & 44.2 & 45.6 & 53.8 & 65.0 & 56.1 & 73.2 & 68.35 & $55.3 \mathrm{~s}$ & 58.14 & 47.44 & 40.3 & 33.6 & 49.5 & 97.06 & $65.6 \mathrm{~T}$ & 73.0 & 47.94 & 47.36 & 68.57 & 76.18 & 80.0 & 42.56 \\
\hline & & 0.6 & 0.5 & B.9 & 4,1 & 2.4 & 0.7 & 0.4 & 5.2 & 0.9 & $0.8 \quad$ & 1.3 & 1.6 & 0.0 & 0.0 & 4.02 & 2.7 & 1.60 & 0.0 & 0.00 & 0.01 & 11.0 & 0.0 \\
\hline & 5 & 0.0 & 0.0 & 0.0 & 0.0 & 0.0 & 0.0 & 0.0 & 0.0 & 0.0 & 0.10 & 0.1 & 0.1 & 0.1 & 0.0 & 0.10 & 0.0 & 0.00 & 0.0 & 0.00 & 0.0 & 0.0 & 0.0 \\
\hline & modallte 6 & 0.0 & 0.0 & 0.0 & 0.0 & 0.0 & 0.0 & 0.0 & 0.0 & 0.0 & 0.0 & 0.0 & 0.0 & 0.0 & 0.0 & $0.0 \quad 0$ & 0.0 & 0.00 & 0.0 & $0.0 \quad 0$ & 0,0 & 0.0 & 0.0 \\
\hline Vartable 9 & modallte I & 26.6 . & 11.8. & 11.1 & 10.5 & 21.7 & 8.3 & 16.1 & 17.22 & 27.32 & 25.23 & 30.6 & 36.53 & 36.1 & $3.6 !$ & 13.34 & 4.71 & 15.01 & 13.83 & 3.4 .6 & 6.91 & 12.9 & 6.7 \\
\hline & $t \in 2$ & 27.9 & 25 & 25.2 & 23.3 & 25.6 & 28.5 & 27.62 & 24.8 .2 & 28.42 & 3 & 30.9 & 28.52 & 23.7 & 9.1 & 13.71 & 15.51 & 17.72 & $23.4 \mathrm{I}$ & 15.41. & 12.38 & 8.72 & 22.8 \\
\hline & 63 & 28.0 & 36.8 & 43 & 43.3 & 35.6 & 38.6 & 34.03 & 36.92 & 28.53 & 30.02 & 26.7 & 26.12 & 28.9 & $\$ 2.6$ & 40.95 & 53.24 & 40.34 & 40.64 & 47.64 & 48.14 & 46.R 3 & 38. \\
\hline & dallite 4 & 17.5 & 26.1 & 20.5 & 23.0 & 17.1 & 24.6 & 22.42 & 21.01 & 15.91 & 19.81 & 11.8 & 8.9 & 11,3 & $34.6:$ & 32.12 & 26.52 & 27.02 & 22.23 & 33.63 & 32.83 & 31.63 & 31.81 \\
\hline fiable 10 & modalite I & 18.8 & 33.7 & 21.2 & 40.8 & 30.0 & 21.1 & 20.21 & 17.31 & 15.52 & 21.81 & 17.4 & 14.2 & 12.0 & 22.02 & 24.63 & 38.4 .4 & 43.94 & 46.74 & 42.93 & 30.11 & 19.93 & 31 \\
\hline & $t 2$ & 29.2 & 32.0 & 40.7 & 32.1 & 27.8 & 19.5 & 20.23 & 34.03 & 33.63 & & 31.8 & 27.9 & 29.6 & 60.46 & $61 . \mathrm{J} 5$ & 52.73 & 34.92 & 28.23 & 36.84 & 49.07 & 76.93 & 39.7 \\
\hline & 6 & 22.3 & 15.0 & 10.5 & 7.3 & 17.4 & 4.0 & 9.61 & 13.61 & 14,41 & 18.72 & 29.2 & 38.6 & 34.6 & 0.5 & 4.3 & 6.22 & 20.02 & 21.73 & 3.44 & 4.31 & 1.6 & $12.8^{\prime}$ \\
\hline & & 26.8 & 17.8 & 27.3 & 18.8 & 22.5 & 55.3 & 49.43 & 34.92 & 28.52 & 21.91 & 18.6 & 14.0 & 18.0. & .16 .1 & 8.52 & 2.7 & 1.13 & $3.3 \mathrm{~J}$ & $16.7 \mathrm{I}$ & 19.90 & 0.3 & 13.11 \\
\hline & modnulte 5 & 2.9 & 1.5 & 0.3 & 1.0 & 2.3 & 0.1 & 0.7 & 0.68 & $8.0 \quad 3$ & 3.02 & 2.9 & 5.3 & 5.8 & 1.0 & 1.40 & 0.0 & 0.10 & 0.0 & 0.20 & 0.6 & 1.3 & 2.5 \\
\hline ariable II & modalite 1 & 61.8 & 50.9 & 61.1 & 65.3 & 70.9 & 71.1 & 66.66 & 64.26 & $62.2 \mathrm{~s}$ & 53.17 & 70.2 & $66.1=$ & 58.8 & 95.5 & 59.08 & 80.76 & 62.68 & 81.76 & 69.27 & 74.18 & 88.1 .4 & 46.97 \\
\hline & 42 & 27.5 & 45.2 & 37.3 & 30.6 & 20.2 & 28.4 & 25.72 & 28.32 & 23.43 & & 17.8 & 15.5 & 16.4 & 3.53 & $31.4 \mathrm{~J}$ & 19.32 & 29.51 & 18.13 & 30.02 & 22.06 & $6.7=$ & 51.91 \\
\hline & 63 & 10.7 & 3.9 & 1.6 & 4.2 & 8.9 & 0.5 & 7.7 & 7.51 & 14.31 & 14.51 & 12.0 & $18.4:$ & 24.8 & 1.0 & 9.70 & 0.0 & 7.90 & 0.10 & 0.83 & 3.95 & 5.2 & 1.11 \\
\hline & modalite 1 & 6.1 & 13.5 & 8.2 & 14,1 & 9,3 & 9.5 & 8.8 & 8.3 & 5.98 & 8.84 & 4.9 & 4.0 & 4.9 & 1.2 & 6.51 & 10.31 & 13.61 & 13.21 & 14.98 & 8.83 & 3.1 & 12.2 \\
\hline & & $2 t .8$ & 32.9 & 20.1 & 14.8 & 10.1 & 19.8 & 19.82 & 26.42 & 21.62 & 28.01 & 15.5 & 12.3 & 13.5 & 21.53 & 37.71 & 15.71 & 15.76 & 6.62 & 22.3 .2 & 24,82 & 22.5 & $43.1 ?$ \\
\hline & 63 & 33.6 & 3 & 56.2 & $\$ 9.1$ & 48.4 & 70.0 & 60.5 & 49.64 & 40.53 & 30.42 & 26.2 & 27.0 & 37.7 & 75.54 & 47,46 & 63.74 & 44,34 & 45,06 & $61.0 \mathrm{~s}$ & 59.06 & 66.33 & 30.6 \\
\hline & modellue 4 & 38.5 & 16.1 & 15.4 & 12.0 & 32.2 & 0.7 & $10.9 \mathrm{I}$ & 15.73 & 31.93 & 32.95 & 53.4 & 56.7 & 43.9 & 1.7 & 8.41 & 10.32 & 26.33 & 35.1 & 1.87 & 7.5 .8 & 8.1 & 14.12 \\
\hline able 13 & modelite 1 & 27.5 & 45.0 & 20.8 & 18.1 & 12.4 & 26.2 & 25.63 & $\overline{34.62}$ & 28.33 & 37.02 & 21.9 & 18.2 & 17.8 & 3.63 & 37.28 & $\overline{8.5} 1$ & 15.96 & 6.12 & $\overline{21.41}$ & 19.91 & 17.7 & 31.5 \\
\hline & & 9 & & & & 36.5 & 13.6 & 14.91 & 13.12 & $23.2 \mathrm{I}$ & & 16.0 & & & 3.9 & 6.74 & 42.44 & 43.34 & & 39.82 & 20.91 & 13.2 & 17.01 \\
\hline & detine 3 & 25.1 & 14.6 & 14,8 & 12.2 & 20.8 & 1.5 & 2.6 & 5.41 & 14.61 & 14.72 & 29.0 & 29.1 & 16.4 & 0.4 & $5.2 !$ & 11.61 & 14.72 & 26.60 & 0.71 & 1.11 & 1.4 & 6.31 \\
\hline & deltat 4 & 34.2 & 21.2 & 30.6 & 210 & 29.8 & 55.1 & 54.24 & 41.33 & 31.52 & 28.93 & 31.5 & 36.13 & 39.8 & 15.8 & 7.55 & 5.1 & 12.81 & 11.91 & 16.71 & 17.51 & 1.0 & 14.6 \\
\hline & modalite 5 & 3.7 & 4.5 & 2.4 & 3.3 & 0.6 & 3.6 & 2.5 & 5.62 & 2.52 & 2.61 & 3.6 & 2.1 & 7.6 & 76.24 & 43.43 & 32.41 & 13.27 & 7.92 & 21.44 & 40.76 & 66.7 & 10.6 \\
\hline tר & modallit 1 & 31.8 & 23.3 & 22.4 & 20.7 & 27.12 & 21.7 & 23.32 & 23.12 & 26.32 & 25.03 & 35.0 & 39.13 & 34.0 & 6.6 & 8.81 & 13.81 & 15.82 & 22.6 & 6.77 & 7.42 & 2.2 & 10.8 \\
\hline & & 18.2 & 14.3 & B.S & 8.7 & 13.5 & 4.3 & 5.8 & 7.01 & $14.8 \mathrm{I}$ & 12 & 20.8 & 17.7 & 12.1 & 1.1 & 6.46 & 6.5 & 9.51 & 14.93 & 3.74 & 4.0 & 2.9 & 3.8 \\
\hline & Le 3 & 5.4 & 9.2 & 4.2 & 2.9 & 2.1 & 5.5 & 5.3 & 6.75 & 5.67 & 7.54 & 4.1 & 3.0 & 2.9 & 0.7 & 6.91 & 1.2 & 3.81 & 1.4 & $4.8 \quad 4$ & 4.8 & 1.6 & 15.0 \\
\hline & dallte 4 & 13.0 & 16.0 & 31.5 & 36.8 & $32.0=$ & 28.8 & 28.52 & 25.32 & 21.31 & 16.11 & 147 & 21.82 & 28.2 & 7.2 & 6.93 & 34.03 & 35.73 & 36.73 & 35.12 & $20.3 ?$ & 2.6 & 14.8 1 \\
\hline & & 6.0 & 8.6 & 13.1 & 14.4 & 11.2 & 6.7 & 6.6 & 6.27 & 7.28 & 8.15 & 5.8 & 3.4 & 3.5 & 1.1 & 6.51 & 10.61 & 14.11 & 12.81 & 12.98 & 8.24 & 4.4 & 10.7 \\
\hline & & 7.7 & 9.2 & 4.0 & 3.7 & 3.7 & 5.5 & 6.2 & 6.58 & 8.09 & 9.76 & 6.6 & 3.8 & 3.6 & 1.1 & 7.50 & 0.3 & 3.51 & 1.3 & 4.94 & 4.82 & 2.3 & 9.0 \\
\hline & $t 7$ & 10.7 & 8.3 & 10.8 & 8.3 & 7.83 & 20.8 & 17.81 & 14.61 & 10.38 & 8.77 & 7.2 & 5.4 & 10.2 & 43.42 & 25.31 & 16.4 & 8.06 & 6.01 & 18.42 & 27.94 & 43.7 & 12.8 \\
\hline & 68 & 5.4 & 8.1 & 3.8 & 3.4 & 1.7 & 4.9 & 4.8 & 7.5 & 4.7 & 6.13 & 3.9 & 3.9 & 4.6 & 38.62 & 27.61 & 17.1 & 8.43 & 3.91 & 11.92 & 21.22 & 29.5 & 10.11 \\
\hline & modallte 9 & 1.8 & 3.1 & 1.6 & 1.2 & 0.8 & 1.8 & 1.8 & 3.01 & 1.92 & 2.61 & 1.9 & 1.8 & 0.8 & 0.2 & 4,0 & 0.1 & 1.10 & 0.41 & 1.61 & 1.45 & 5.9 & 3.0 \\
\hline & adallte 1 & 0.2 & 1.3 & 3.4 & 0.6 & 0.8 & 1.8 & 1.5 & $\begin{array}{ll}5.6 \quad 1 \\
\end{array}$ & $\begin{array}{ll}1.8 \\
\end{array}$ & 1.81 & 1.1 & 2.3 & 3.6 & 0.0 & 0.0 & 3.4 & 0,0 & 0.0 & 0.7 & 1,00 & 0.2 & 0.0 \\
\hline & & 4.2 & & I. & 1.6 & 3.1 & 0. & 2. & 6 & 6.85 & 5. & & & 3.1 & & 42 & 0.0 & 3.0 & 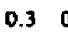 & & 2.32 & & 4.4 \\
\hline & & 1.4 & 0.5 & 0.5 & 0.5 & 1.0 & 0.0 & 0.6 & 1.22 & 2.32 & 2.12 & 2.1 & 2.7 & 1.3 & 0.3 & 1.40 & 0.0 & 0.30 & 0.1 & 0.3 & 0.80 & 0.8 & 1.5 \\
\hline & dallite 4 & 69.9 & 68.6 & 80.9 & 84.9 & 82.5 & 81.9 & 80.06 & 65.46 & 63.06 & 65.36 & 67.7 & 69.2 & 67.7 & 19.83 & 32.06 & 60.57 & 73.58 & 80.26 & 66.54 & 45,32 & 23.1 & 52.6 \\
\hline & odallite 5 & 24.4 & & 14.1 & 12.3 & 12.6 & 16.3 & 15.82 & 24.12 & 26.22 & 25.02 & 23.1 & 24.1 & 24.2 & 79.06 & 62.43 & 36.12 & 25.2 I & 19.43 & 31.85 & 50.87 & 73.5 & 41.5 \\
\hline
\end{tabular}




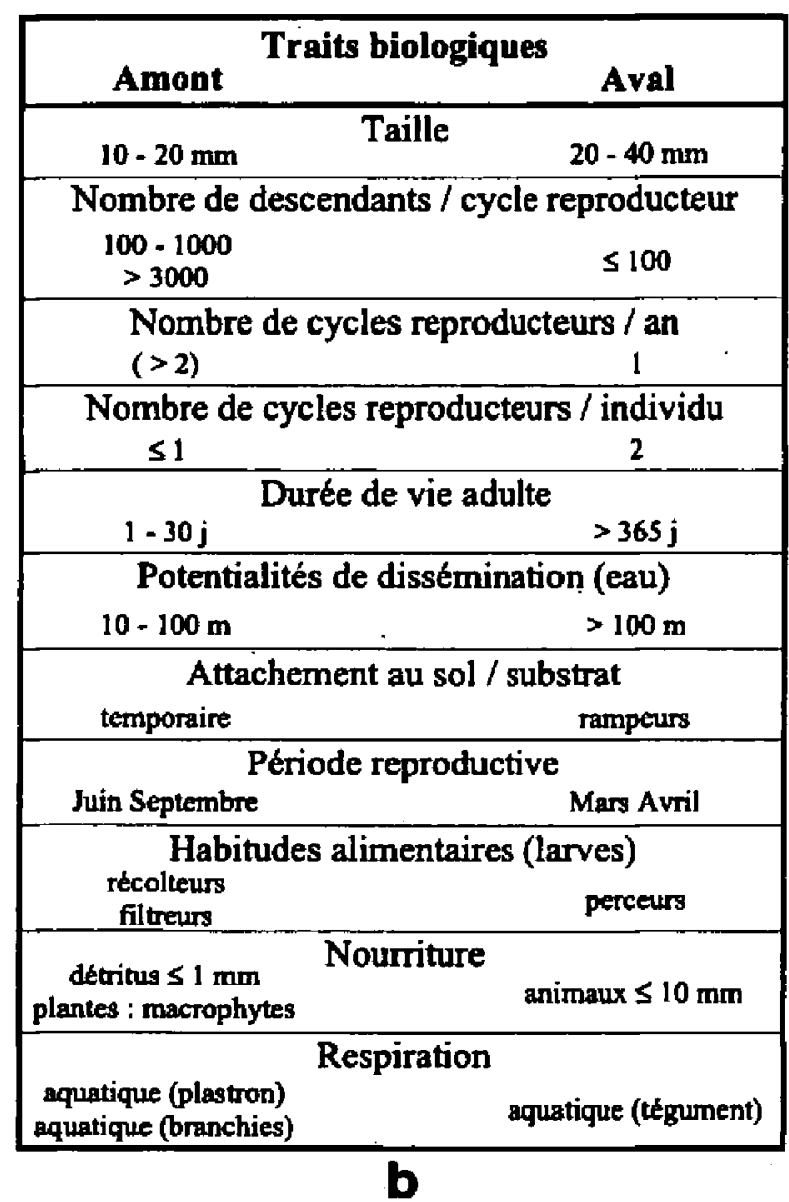

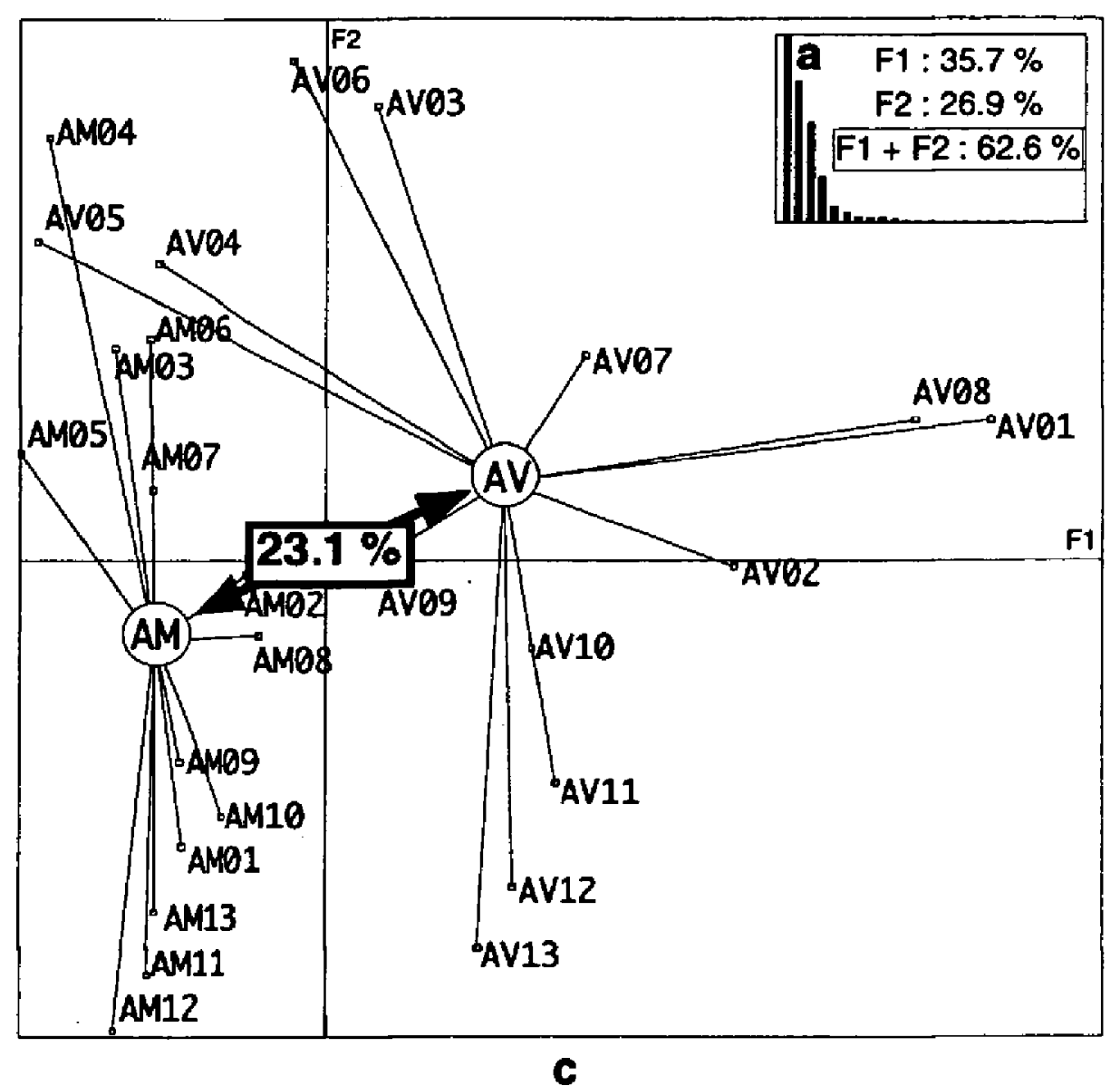

Fig. 3. Analyse des Correspondances Floues réalisée sur le tableau obtenu par pondération des traits biologiques par les abondances spécifiques. a : graphique des valeurs propres. b : modalités des traits biologiques significativement différentes en amont et en aval, suite à un test de la somme des rangs $(p<0.05)$; entre parenthèses, résultat d'un test des rangs signés $(p=0.1)$. c : carte factorielle des échantillons $(A M$ et $A V$ sont les centres de gravité des nuages des échantillons amont et aval, numérotés de 01 à 13).

Fig. 3. Fuzzy Correspondence Analysis performed on the combined array of biological traits and species abundances. a : graphic of eigenvalues. b : modalities of biological traits significantly different upstream and downstream, obtained with a rank sum test $(\mathrm{p}<0.05)$; in parentheses, result of a signed rank test $(p=0.1)$. $c$ : factor map of samples (AM and AV are gravity centers of the two stars of upstream and downstream samples, numbered 01 to 13 ).

La comparaison des deux approches (tableau des effectifs $=$ Tableau 2 ; tableau des traits biologiques $=$ Tàbleau 3) a été réalisée à l'aide de l'analyse de variance à deux facteurs contrôlés, le premier étant le type d'àpproche, et le deuxième étant l'effort d'échantillonnage (les prélèvements lentiques seulement, lotiques seulement, ou la somme des deux). Elle a permis de montrer, sur la base des distances séparant les stations à chaque date, que l'approche par les traits biologiques est plus discriminante $(p<0,01)$. Cependant, pour les deux approches, la variabilité saisonnière ne joue pas un rôle significatif. De même, l'effort d'échantillonnage n'a pas un effet significatif sur le pouvoir de discrimination.

\section{Discussion}

\subsection{Comparaison des traits biologiques avec les autres approches}

L'approche basée sur l'étude des traits biologiques, appelée ici «approche fonctionnelle», sépare les sta- tions amont des stations aval de façon plus tranchée que la prise en considération des variables physicochimiques : en effet, si le premier axe factoriel de l'ACPN des variables physico-chimiques peut être interprété comme l'axe amont-aval, il est en fait essentiellement défini par trois échantillons estivaux aval, AV05, AV06, AV07, qui permettent le décalage du centre de gravité de l'ellipse vers la gauche, et donc constituent l'essentiel des 3,1\% de séparation (Fig. 1). En été, les conditions de basses eaux font que l'effluent de la station d'épuration devient plus concentré, entraînant de fortes valeurs pour certaines variables.

En revanche, l'approche basée sur les effectifs des taxons, appelée ici «approche biocénotique», et la précédente approche (fonctionnelle) présentent des points communs : elles permettent de discriminer les situations amont des situations aval, et elles ne sont pas influencées par l'effort d'échantillonnage et la variabilité saisonnière. Toutefois l'analyse de variance a montré que l'approche fonctionnelle est significativement 
plus discriminante que l'approche biocénotique. En effet, l'approche fonctionnelle repose sur les abondances taxinomiques trouvées dans les prélèvements, comme l'approche biocénotique, mais ces abondances sont combinées avec une information biologique (les scores) caractérisant chaque taxon trouvé. Ainsi, l'information présente dans le tableau qui en résulte est plus riche que les abondances seules, donc il n'est pas illogique que la discrimination entre les stations amont et aval soit plus importante.

\subsection{Signification des traits biologiques et relations avec les concepts théoriques}

Certains auteurs tels Southwood (1988) et Townsend \& Hildrew (1994), déclarent que l'habitat est le cadre dans lequel l'évolution forge les caractéristiques des traits de vie des organismes. Dans leurs études, ils développent un modèle d'habitat rivulaire, et font des prédictions concernant les traits des espèces attendus dans certains types d'habitats appartenant au modèle. C'est ainsi qu'ils prédisent que les habitats à forte variabilité temporelle doivent abriter des espèces à durée de vie courte et à croissance populationnelle élevée, donc caractéristiques de la résilience. Au contraire, les habitats plus constants doivent abriter des espèces à durée de vie longue, de grande taille et pouvant avoir plusieurs épisodes de reproduction, donc des caractéristiques de résistance. Leur argument est fondé sur le même raisonnement que celui du concept r/K (Pianka 1970).

La taille, le nombre de descendants par cycle reproducteur, le nombre de cycles reproducteurs par an, le nombre de cycles reproducteurs par individu, la durée de vie adulte, les pótentialités de dissémination (dans l'eau) et l'attachement au sol ou au substrat, sont des variables significatives dans notre étude et discutées par Townsend \& Hildrew (1994). Dans nos échantillons amont (Fig. 3), les modalités observées pour toutes ces variables, sauf la dernière, confirment précisément les prédictions de ces deux auteurs concernant un habitat «temporellement variable et spatialement uniforme». Ces modalités sont considérées comme étant caractéristiques de la «résilience». Au contraire, nos résultats pour les échantillons aval correspondent, selon ces mêmes auteurs, à leur définition de la «résistance», c'est-à-dire caractérisant des habitats constants.

La période de reproduction est située entre juin et septembre, pour ce qui est des échantillons amont, ce qui est tout à fait normal pour des communautés benthiques, puisque l'augmentation de la température de l'eau est connue pour avancer l'émergence, et inverse- ment (Williams \& Feltmate 1992). Cependant, pour les échantillons aval, il semble que la biocénose tende à éviter la saison estivale pour sa reproduction, ce qui peut s'expliquer par les conditions physico-chimiques particulièrement difficiles à cette époque (Fig. 1 : AV05, AV06, AV07). Ainsi, la reproduction a-t-elle lieu au printemps.

Les habitudes alimentaires et la nourriture sont deux variables inséparables. Selon Southwood (1988), repris dans Williams \& Feltmate (1992), les échantillons amont possèdent des modalités caractéristiques d'un type d'habitat où l'adversité est très faible (ou par comparaison la productivité est très élevée), tandis que les échantillons aval possèdent des modalités caractéristiques d'un type d'habitat où l'adversité est très élevée (ou par comparaison la productivité est très faible).

Dans les échantillons amont, la respiration est assurée soit par des plastrons, soit par des branchies. Les plastrons permettent aux insectes d'utiliser l'oxygène atmosphérique, mais sont dépendants d'autres facteurs tels la température de l'eau, la teneur en oxygène dissous, la pollution organique (Williams \& Feltmate 1992). Les branchies sont généralement plus fréquentes dans les eaux courantes ou dans les eaux stagnantes bien aérées, mais l'efficacité de ce système respiratoire est améliorée par le comportement (par ex. en augmentant la circulation de l'eau autour des branchies), ce qui confère aux insectes une meilleure tolérance aux faibles teneurs en oxygène (Williams \& Feltmate 1992). Selon ces auteurs, ce système serait particulièrement avanțageux pour les espèces vivant dans des habitats où les niveaux en oxygène varient selon les saisons. Ainsi, ces deux systèmes respiratoires peuvent être perçus comme un moyen de supporter des conditions difficiles, ce qui est une caractéristique de «résilience». Au contraire, la respiration tégumentaire ne fournit une quantité d'oxygène suffisante que pour des invertébrés relativement inactifs, même si le système est rendu plus performant par l'utilisation d'un vecteur d'oxygène plus efficace dans l'hémolymphe (Williams \& Feltmate 1992) : ceci peut être interprété comme une caractéristique de «résistance».

\section{Conclusion}

Nos résultats ont montré que la situation amont, c'est-à-dire légèrement polluée, comporte une biocénose dont les caractères biologiques sont typiquement rencontrés dans des environnements où la variabilité est forte, l'adversité est faible, et la stratégie biologique est la résilience, ou sélection r (Pianka 1970, Greenslade 1983, Southwood 1977, 1988). 
Inversement, la situation aval, fortement polluée, comporte une biocénose dont les caractères biologiques sont typiquement rencontrés dans des environnements où la variabilité est faible, l'adversité est forte, et la stratégie biologique est la résistance, ou sélection A.

Ainsi avec cette étude, nous pouvons d'ores et déjà affirmer que les traits biologiques, c'est-à-dire le codage des connaissances concernant la biologie des macroinvertébrés benthiques, représentent un outil susceptible de mettre en évidence l'effet d'une pollution. Cette approche est dans le cas de la Chalaronne plus discriminante qu'une approche physico-chimique classique, en raison du côté intégrateur que possède l'information biologique. Mais cette approche est aussi plus discriminante que l'approche biocénotique, parce que l'information analysée est plus riche que l'étude des seules abondances.

Ce travail nécessite d'être poursuivi en développant d'autres applications, essentiellement sur des types bien différenciés de pollutions, afin de révéler l'évolution des traits biologiques face aux altérations. Mais cette méthode doit aussi être testée sur des situations exemptes de pollution, afin d'affirmer ou d'infirmer les prédictions concernant la sélection $\mathrm{K}$, caractéristique d'un environnement où la variabilité et l'adversitế sont faibles.

\section{Remerciements}

Les auteurs remercient Anne Kosmala (Cemagref, Lyon) qui est l'initiatrice de cette étude d'impact d'une station d'épuration sur la faune in situ ; le laboratoire d' 'Ecologie des Eaux Douces et des Grands Fleuves» de l'Université de Lyon I pour la mise à disposition de leurs connaissances des stratégies biologiques des macroinvertébrés benthiques et pour leur assistance au niveau du traitement des données écologiques ; le Cemagref et le GIP Hydrosystèmes (Programme : Evaluation de l'état de santé écologique des hydrosystèmes par l'utilisation des variables biologiques) pour leur soutien financier. Les auteurs remercient également deux lecteurs anonymes pour leurs remarques et les améliorations qu'ils ont apporté au manuscrit.

\section{Travaux cités}

AFNOR 1992. - Détermination de l'indice biologique global normalise (IBGN). Association Française de Normalisation : NF T 90-350: 9 p.

Benzécri J.P. 1973a. - L'analyse des données. I : la taxinomie. Dunod, Paris : $615 \mathrm{p}$.

Benzécri J.P. 1973b. - L'analyse des données. II : l'analyse des correspondances. Dunod, Paris : $619 \mathrm{p}$.

Chapman D. 1992. - Water quality assessment. Chapman \& Hall, London : 585 p.
Chessel D. \& Doledec S. 1992. - ADE Software (Version 3.6). Multivariate analyses and graphical display for environmental data. User's manual. URA CNRS 1451 'Ecologie des Eaux Douces et des Grands Fleuves', University of Lyon I, Villeurbanne : $127 \mathrm{p}$.

Chevenet F., Dolédec S. \& Chessel D. 1994. - A fuzzy coding approach for the analysis of long-term ecological data. Freshwater Biol., 31 : 295-309.

Dolédec S. \& Chessel D. 1991. - Recent developments in linear ordination methods for environmental sciences. Advances in Ecology, $1: 133-155$.

Dolédec S. \& Statzner B. 1994. - Theoretical habitat templets, species traits, and species richness : 548 plant and animal species in the Upper Rhône river and its floodplain. Freshwater Biol, 31 : 523-538.

Greenslade P.J.M. 1983. - Adversity selection and the habitat templet. Amer. Natur., 122 : 352-365.

Hotelling H. 1933. - Analysis of a complex of statistical variables into principal components. J. Educat. Psychol., 24 : 498-520.

Hynes H.B.N. 1963. - The biology of polluted waters. Liverpool University Press, Liverpool : $191 \mathrm{p}$.

Lafont M. 1989. - Contribution à la gestion des eaux continentales : utilisation des oligochètes comme descripteurs de l'état biologique et du degré de pollution des eaux et des sédiments. Thèse de Doctorat d'Etat, Univ. Lyon I : 311 p.

Lebart L., Morineau A. \& Piron M. 1995. - Statistique exploratoire multidimensionnelle. Dunod, Paris : $440 \mathrm{p}$.

Macan T.T. 1974. - Running water. Mitt. Internat. Verein. Limnol., 20:301-321.

Mason C.F. 1981. - Biology of freshwater pollution. Longman, London : $250 \mathrm{p}$.

Pianka E.R. 1970. — On r- and K- selection. Amer. Natur., 104 : $592-$ 597.

Southwood T.R.E. 1977. - Habitat, the templet for ecological strategies? J. Anim. Ecol., $46:$ 37-365.

Southwood T.R.E. 1988. - Tactics, strategies and templets. Oikos, $52: 3-18$.

StatSoft Inc. 1997. - STATISTICA for Macintosh [Computer program manual]. StatSoft, Inc., 2300 East 14th Street, Tulsa, OK 74104, USA, phone: (918) 749-1119, fax: (918) 749-2217, email: info@statsoft.com,WEB: http://www.statsoft.com.

Statzner B., Resh V.H. \& Dolédec S. (eds.) 1994a:- Ecology of the Upper Rhône River: : a test of habitat templet theories. Freshwater Biol., 31 : 253-556.

Statzner B., Resh V.H. \& Roux A.L. 1994b. - The synthesis of long-term ecological research in the context of concurrently developed ecological theory : design of a research strategy for the Upper Rhône River and its floodplain. Freshwater Biol., 31 : 253263.

Townsend C.R. \& Hildrew A.G. 1994. - Species traits in relation to a habitat templet for river systems. Freshwater Biol., $31: 265$ 275.

Tufféry G. \& Verneaux J. 1967. — Méthode de détermination de la qualité biologique des eaux courantes. Trav. Sect. P. et P., Cerafer : $23 \mathrm{p}$.

Washington H.G. 1984. — Diversity, biotic and similarity indices. A review with special relevance to aquatic ecosystems. Water Res., $18: 653-694$.

Williams D.D. \& Feltmate B.W. 1992. - Aquatic insects. C.A.B. International, Wallingford : $358 \mathrm{p}$.

Woodiwiss F.S. 1964. - The biological system of stream classification used by the Trent River Board. Chem. and Ind., $11: 443-447$. 